\title{
The Relationship between Students' Perceptions of Parenting Styles and their University Life Adjustment
}

\author{
Said Aldhafri* \\ The Reseach Council \& Sultan Qaboos University, Sultanate of Oman
}

Received: 19/5/2016

Accepted: 26/6/2016

\begin{abstract}
The current study examines the association between university students' perceptions of parenting styles and their university life adjustment. Data were collected from Sultan Qaboos University (SQU) in Oman. The sample consisted of 2562 undergraduate students (59\% were females) from different colleges and study years. The participants responded to two questionnaires. The first questionnaire examined students' perceptions of three parenting styles: authoritative (7 items), authoritarian (7 items), and permissive (6 items). The university life adjustment was examined by a questionnaire that focused on psychological (10 items) and non-psychological (5 items) problems that students face during university years. Both measures were found to be stable and reliable. The findings showed that college students reported high levels of authoritative and authoritarian parenting styles, low levels of permissive style, and low psychological and non-psychological problems. Additionally, results of two separate regression analyses indicated that students' psychological problems were predicted positively by authoritarian mother and negatively by authoritative mother and authoritative father; the non-psychological problems were predicted negatively by authoritative father and positively by authoritarian mother and permissive father. Findings were discussed within Omani cultural context.
\end{abstract}

Keywords: University students' perceptions, parenting styles, university life adjustment.

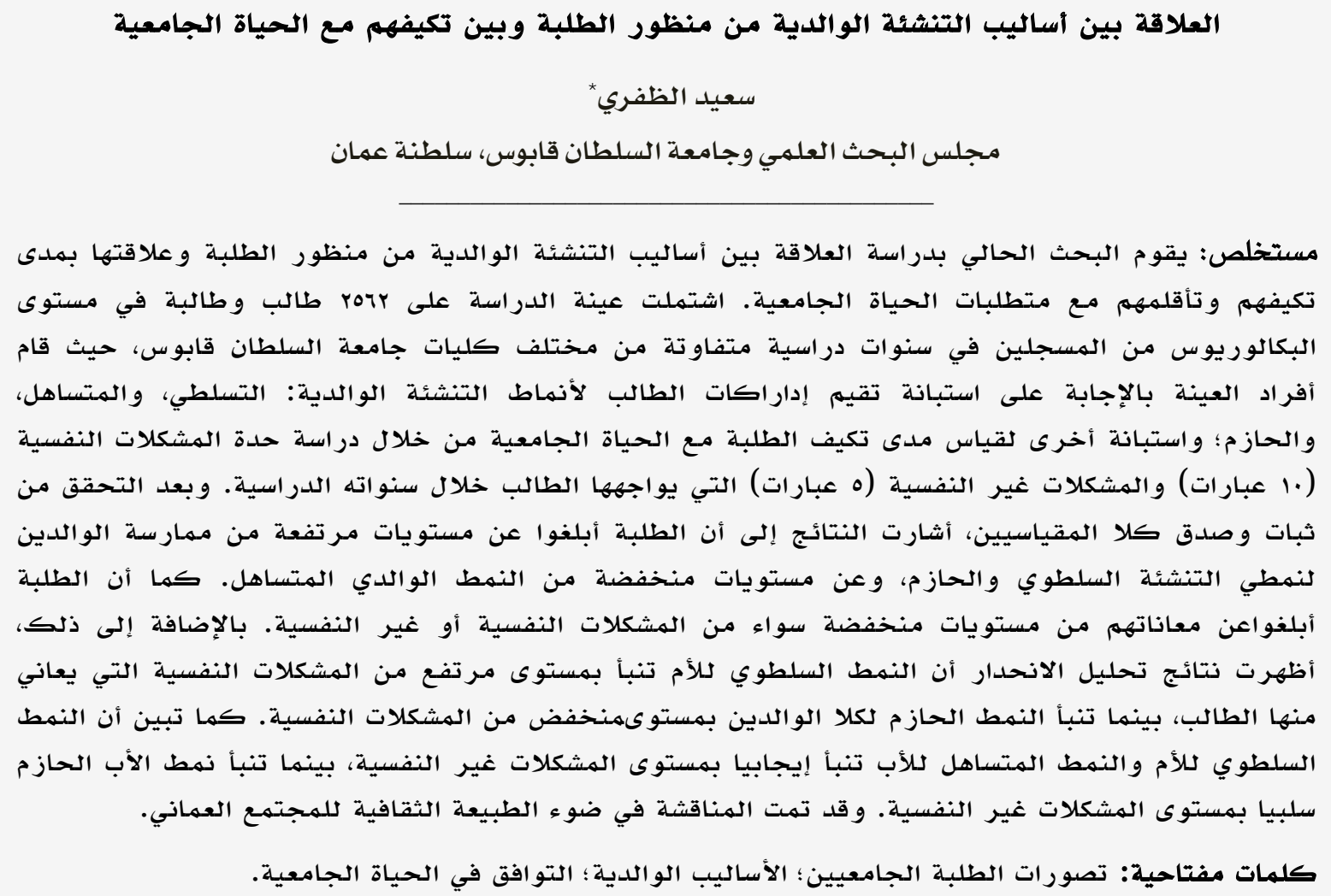

*saidaldhafri@gmail.com 
University students are likely to face different challenges during their academic life in higher education experience (Al-Darmaki, 2003; Benton, Robertson, Tseng, Newton, \& Benton, 2003). Adjustment difficulties had always been the main focus of many researchers worldwide; and problems relevant to students' academic, personal, psychological, professional, and social life and well-being have been identified either in the Western literature (e.g. Love \& Thomas, 2014) or in the Eastern literature (e.g. Al-Darmaki, 2011). Moreover, research indicated a noticeable increase in university and college students' problems in the recent decades compared to the past (e.g. Benton et al., 2003). Such needs, problems, and troubles raised legitimate concerns and were the focus of many investigators who aimed to make the college experience a smooth and peaceful one for students. A plethora of research has demonstrated that parenting styles are pivotal to university students' adjustment (Baldwin, McIntyre, \& Hardaway, 2007; Joshi, Ferris, Otto, \& Regan, 2003; Love \& Thomas, 2014), and a growing number of studies have focused on understanding university students' life adjustment by examining possible predictors that may explain the meaningful trajectories of university problems. The current study focuses on how students' perceptions of parenting styles may relate to the levels of problems they face during university years.

Family related matters are considered distracters that worry university students. Since most university students live away from home to attend university, this might arouse students' concern about their family members' well-being. In addition, attitudes and beliefs' conflict in the parent-adolescent relationship regarding students' future plans can arouse the sense of discomfort within students that might contribute to their maladjustment and overall unhealthy well-being (Cheung, \& Nguyen, 2009). In line with these kinds of family relevant problems, college students worry about their future marital status, such as finding the right spouse and the financial stability to afford wedding expanses (Al-Darmaki, 2011; Reynolds, 2013).

Perceived parenting styles. Parenting style is a construct that refers to the parental pattern of practices, tactics, strategies, and behaviors that are adopted and used to educate children, manipulate their behavior, and interact with them in general (Chenung \& Nguyen, 2009). It has also been defined as a socialization approach adopted by parents to teach their children norms, values, behavior, and necessary social skills. Baumrind (1991) classified parenting styles based on the levels of two characteristics, responsiveness and demandedness. "Responsiveness" is described as warmth or supportiveness, which refers to the extent to which parents intentionally cultivate their children's individuality, self-regulation strategies, and assertiveness by giving attention, providing support, and responding to particular concerns, needs, and demands. Parental demandingness is expressed as the behavioral control and the expectations put on their children to become a part of the family by the parents' rules, supervision, and disciplinary practices." (Love \& Thomas, 2014, p. 140). Four styles can be described based on the combination of responsinvess and demandingness. These include the permissive, the authoritative, the authoritarian, and the neglectful parenting style. Each style is characterized by different levels of exhibited responsiveness and control by parents towards their children (Rinaldi \& Howe, 2012).

The authoritarian parenting style is characterized by strictness of the parents. An authoritarian parent would exhibit a high level of control and demandingness. In addition, authoritarian parents tend to be thrifty in showing emotionality and responsiveness towards their children (Maddahi et al., 2012). A clear conduct of behavior is usually stated between authoritarian parents and their children. This conduct is monitored and controlled by reward and punishment strategies in most of the cases. Parent who adopt this style expect their children to carry out their instructions without negotiation or further inquiries (Baumrind, 1991).

On the other hand, permissive parenting is framed by more flexibility and less strictness in comparison to the authoritarian parenting style. Permissive parents are generally less demanding and show higher levels of responsiveness towards their children's behaviors. They allow a platform for the child to express and feel comfortable with discussion and negotiation. No clear behavior conduct is outlined between parents and children in this style and so children are permitted a grand opportunity to practice self-regulation and discovery (Conrade \& Ho, 2001). 
Finally, authoritative parenting is a median version of parenting styles that has characters from both the previous discussed styles. Authoritative parents keep a balance between the levels of demandingness and responsiveness that they exhibit towards their children (Baumrind, 1991). Strategies from both the aforementioned styles might be adopted and implemented according to the situation. Parents who follow this parenting style tend to put standards and monitor their children behavior in a supportive rather than aggressive way. Authoritative parents are referred to as "assertive" parents who seek positive outcomes of their child behavior without being "restrictive" (Maddahi et al., 2012).

Seldom, parents adopt a fourth type of parenting referred to as the "neglectful" parenting style. Parents following this style usually fail to attend to their parental responsibility, as they exhibit disinterest in being involved in their childrens' behaviors. Neglectful parents are neither responsive nor demanding (Baumrind, 2005).

University life adjustment. College students are likely to undergo various challenges and face different problems. Uncertainty about selecting the right major and lack of clear future plan and guideline could irritate students and raise a sense of anxiety and motivate them to achieve. Accordingly, students may experience low self-efficacy and start to disbelieve in their abilities and skills (Chandler \& Gallagher, 1996; Love \& Thomas, 2014; Pedrelli et al., 2013; Reynolds, 2013). Procrastination and difficulty to properly manage time are also major threats that directly affect students' academic performance quality (Al-Daramki, 2011). In addition to study and academic concerns, some students may find coping with university life a major challenge due to their lack of some necessary skills like communication and language skills. Shyness is one reason that serves as a barrier between students and peaceful coping with university life. Also, the foreign languages that are used in the higher education environment can shake students' confidence in their abilities to communicate well with others. Shyness and attempting to avoid linguistic mistakes while contacting with others might drive students to keep a distance from other members in the college community (Asberg, 2013). Furthermore, college students' psychological and mental health is also more likely to be affected by students' engagement in failure romantic relationships (Al-Darmaki, 2011; Chandler \& Gallagher, 1996).

One of the dominant problems that students may face in their higher education stage is the continuous concern about their future employment. Students may grow suspicious about the availability of suitable jobs in the labor market that would fit their major. Also, the competition over limited vacancies and opportunities makes students worried about their success chances in the future (AlDarmaki, 2011; Chandler \& Gallagher, 1996).

Health and physical problems can also contribute to making college experience a tough one for students. Chronic diseases such as anemia and diabetes might require students to miss many of their classes, which could result on failing some courses. Moreover, college students are likely to experience diet and sleep disorders, which influence their well-being and affect their attention in the classroom $\mathrm{Al}$ Darmaki, 2011; Chandler \& Gallagher, 1996; Wagner \& Rhee, 2013).

Perceived parenting and university adjustment. Cumulative research demonstrated that positive parenting and continuous involvement and support by parents resulted in positive outcomes for the children, while negative characteristics of parenting, such as poor supervision and inconsistent discipline, are associated with higher levels of children's behavioral, social, psychological, and academic problems (e.g. Gryckowski, Jordan, \& Mercer, 2010). The current study examines the role of university students' perceptions of their parenting styles and how these perceptions influence the levels of students' university adjustment.

Previous research attempted to address the issue of college students' problems and investigated its association with different variables (Al-Darmaki, 2011; Asberg, 2013). However, limited research shed light on perceived parenting styles and college experience quality (e.g. Estep \& Oslon, 2011; Baldwin, Hardaway, \& McIntyre, 2007). Research demonstrated the existence of parental influence on adult students' lifves; for example, Aldhafri and AlHarthy (2016) found that authoritarian parenting styles predicted university students' foreclosure academic identities. The researchers 
reported positive relationship between an authoritative parenting style and achievement academic identity. While the diffusion dimension of academic identity was influenced positively by both authoritarian and permissive parenting styles, none of the parenting styles correlated significantly or predicted students' moratorium levels. Love and Thomas (2014) found that parenting practices explained 25\% of the variance in self-esteem and $8 \%$ of the variance in emotional well-being in a sample of college level students (using Marcia's conceptual framework of identity (foreclosure, diffusion, moratorium, and achievement) and applying it to the academic context).

Considering the most prominent parenting styles, i.e. authoritarian, authoritative, permissive, and neglectful, the authoritarian parenting style was proved to restrict adult students' stability and to have an unpleasant influence on students' outcomes. A study by Love and Thomas (2014) found that authoritarian parenting associated with a high level of emotional distress among college students. Estep and Olson (2011) found also that the parenting dimension of strictness control has a negative association with college students' tendencies to get involved in inappropriate academic and romantic practices among a sample of 109 students recruited from the University of Texas. Gracia and Gracia (2009) concluded that offspring of authoritarian parents in the Spanish context scored lower than their peers from permissive and authoritative families in different important dimensions, like self-esteem, psychological maladjustment, personal competence, and problem-solving behavior.

On the other hand, permissive parenting, has been found to be a significant predictor of optimum youth outcomes. Aldhafri (2011) found father permissive parenting style connected with increase in adolescents' school and health problems. Love and Thomas (2014) found that permissive parenting resulted in low scores in self-esteem and emotional well-being in a sample of college students in the Eastern and Southern United States. However, and opposite to the aforementioned results, Gracia and Gracia, (2009) studied the relationship between adolescents' perceived parenting style and their overall well-being outcomes among almost one and a half thousand adolescents in the Spanish context. Their results indicated that better outcomes were achieved by those who perceived their parents to be permissive.
Social competence performance was also demonstrated to be positively associated with permissive and authoritative maternal parenting style in a sample of 737 Iranian adolescent girls (Ardabili, Kazemi, \& Solokian, 2010).

Combined involvement with strictness (resembling the authoritative parenting style) was found optimally the most influential parenting style in predicting positive youth outcomes. Baldwin, Hardaway, and McIntyre (2007) found that authoritative parenting style correlated positively with college students' levels of optimism. Love and Thomas (2014) found that authoritative parenting is a positive predictor of self-esteem and emotional wellbeing among college level students, unlike permissive and authoritarian parenting styles. Similar findings by other research worldwide demonstrated that authoritative parenting is associated with better emotional, social, and psychological competence and adjustment levels, which, as a consequence, allow students to deal with problems and life challenges more positively (e.g. Grundman, 2011; Ishak et al., 2012; Mckinney, Milone, \& Renk, 2011; Mckinney \& Renk, 2008; Strand \& Bradt, 1999).

Compared to what is known about school students' parenting styles, little is known about university students' perceptions of parenting styles and how these perceptions may influence students' university outcomes. Hence, the current paper aims to examine the relationship between perceived parenting style and problems faced by students at Sultan Qaboos University (SQU). If connections between parenting styles and students' university outcomes are proved, possible counseling interventions can be designed for first-year students to help control for the effects of their perceptions of parenting styles on their university adjustment. 


\section{Method}

\section{Sample}

The current sample consisted of 2562 undergraduate students (59\% were females) from Sultan Qaboos University in Oman. The students came from different colleges with the majority of them coming from colleges of economic and political sciences $(23.1 \%)$, art and social sciences $(23.3 \%)$, engineering $(13.2 \%)$, and education $(14.1 \%)$. They are in their first $(16 \%)$, second $(18.7 \%)$, third $(21 \%)$, fourth $(25.4 \%)$, or fifth year and above (18.9\%). These students originally reside in different parts of the country, with the majority coming from Aldakhliya (20.1\%), Muscat (17.2\%), North Batina (17.2\%), and South Batina (12.8\%). During the academic year, most of the sample reside on campus $(47.2 \%)$, rent with friends $(28 \%)$, rent alone $(2.3 \%)$, or live with their family $(22.6 \%)$. Four percent of the participants reported grade point an average (GPA) of less than 2.00; $26 \%$ reported GPA between 2.00$2.49 ; 40.5 \%$ were between $2.50-2.99$; and the rest $(29.6 \%)$ scored 3.00 or above. Using a convenient sample approach, the students were invited to participate in the study by a group of research assistants who visited available classes. Data collection took place during spring 2015. Participation was voluntary and participants did not get anything for their participation. All students who were attending classes during data collection agreed to participate in the study. The students were assured of confidentiality and were asked not to put any identification information on the instruments. Prior to data collection, the study was approved by the Sultan Qaboos University Institutional Review Board for the use of $\mathrm{Hu}-$ man Subjects.

\section{Instruments}

The research methodology employed in this study is a quantitative methodology of survey research. The survey research included two self-report questionnaires as well as a demographic survey created by the current researcher. The first questionnaire examined students' perceptions of the parenting styles they have experienced, and the second questionnaire related to their perception of their adjustment to university life. The two variables were defined operationally based on participants' scores in the two questionnaires used in the current investigation.
The first measure was the short version of the Parental Authority Questionnaire (PAQ, Buri, 1991). This short version consisted of 20 items and examined the same three dimensions of the long version of the PAQ that included authoritarian, authoritative, and permissive parenting styles. Alkharusi, Aldhafri, Kazem, Alzubiadi, and Al-Bahrani (2011) examined the validity and reliability of the short version and reported adequate evidences using a sample of Omani school students. Based on previous studies' recommendation (e.g., Aldhafri, 2011), mother's parenting styles were examined separately from father's parenting styles. Using the current data, for the mother's parenting style scale, a pre-specified threefactor solution using exploratory factor analysis (with promax rotation and maximum likelihood extraction) provided support for the structural validity of the PAQ $(35.25 \%$ of variance accounted for). All items loaded on their respective dimensions with loadings above 0.30 . Acceptable reliability coefficients were obtained for the three dimensions $(\alpha=0.73$, 0.80 , \& 0.66$)$, respectively. For the father's parenting styles scale, similar results were found. The three-factor solution explained $35.69 \%$ of variance and all items loaded in their theoretical dimensions with loading values above 0.30 . Reliability coefficients of $0.74,0.82$, and 0.60 were obtained for the three parenting styles, respectively. Because the PAQ is a five-point Likert scale, mean scores range from 1 to 5 , with 1 indicating little recognition by the respondent of the parenting style of each parent and 5 indicating total recognition by the respondent of the parenting style of each parent. Possible scores can range from 20 to 100 with high scores indicating high levels of the use of parenting styles.

For the second measure that assessed university students' life adjustment, the researcher constructed a new measure for this purpose. The University Life Adjustment Scale (ULAS) consisted of 15 types of problems that are dominant in the university context. The participants were instructed to report to what extent they faced each type of the 15 problems using a 5-point Likert scale that ranges from 5 (high degree) to 1 (I do not face this problem). The measure was subjected to an exploratory factor analysis using promax rotation with a maximum likelihood extraction methodology. The results showed a two-factor solution that ac- 
counted for $44.56 \%$ of variance. The first factor included psychological problems such as low motivation, low confidence, shyness, maladaptation, and loneliness. This factor with 10 items showed good reliability coefficient $(\alpha=$ 0.88 ). The second factor included nonpsychological problems that relate to family problems, eating disorder, sleeping disorder, and health problems (5 items, $a=0.76$ ). The two questionnaires can be obtained from the author.

\section{Results}

Data from the current study were processed and analyzed by using Statistical Package for Social Science (SPSS). Two statistical procedures, descriptive analysis and inferential statistical analysis, were used for the data analysis. Data were first screened for outliers and no threat was identified. Descriptive statistics of students' levels of the two types of university problems (psychological problems and nonpsychological problems) showed statistically low levels of the actual means compared to the theoretical means. The results also show lower levels of permissive parenting styles for both the father and the mother. In contrast, the students reported statistically higher levels of both authoritative and authoritarian parenting styles for the two parents. Table 1 displays these findings.

Prior to examining the predictive role of parenting styles, simple correlation coefficients were first examined among the study variables using Pearson coefficients (see Table 2). The results show that the six parenting styles (three for each parent) correlated significantly with the two types of university students' problems. While authoritative parenting style correlated negatively with the levels of students' problems, both authoritarian and permissive styles correlated positively.
Table 1

Means, Standard Deviations, and the One-Sample t-test on the Model's Variables $(\mathrm{N}=\mathbf{2 5 6 2})$

\begin{tabular}{|c|c|c|c|}
\hline Variable & M & SD & $\mathrm{t}$ \\
\hline Authoritarian mother & 3.422 & 0.722 & 28.958 \\
\hline Authoritative mother & 3.941 & 0.643 & 72.418 \\
\hline Permissive mother & 2.933 & 0.747 & -4.374 \\
\hline Authoritarian father & 3.446 & 0.746 & 30.262 \\
\hline Authoritative father & 3.753 & 0.742 & 51.299 \\
\hline Permissive father & 2.921 & 0.706 & -5.610 \\
\hline Psychological problems & 2.282 & 0.757 & -47.406 \\
\hline $\begin{array}{l}\text { Non-Psychological } \\
\text { problems }\end{array}$ & 1.901 & 0.811 & -67.806 \\
\hline
\end{tabular}

Two regression equations were run to examine parenting styles as a predictor of the university students' problems (see Table 3). In the first equation, psychological problems were predicted using the set of six parenting styles. The model of the six parenting styles explained only $4 \%$ of variance in the psychological problems. Three parenting styles were significant; these include the authoritative father $(t=-4.55$, $p<0.001)$, the authoritarian mother $(t=3.59, p<$ $0.001)$, and the authoritative mother $(t=-2.98$, $p<0.01$ ). The other three variables were not significant. These were the authoritarian father, the permissive father, and the permissive mother.

The second equation dealt with prediction of the second dimension of students' problems (i.e., non-psychological problems) using the six dimensions of parenting styles. The results showed that the model explained only $5 \%$ of variance. Similar to the first regression equation, the authoritative father $(t=-5.12, p<$ $0.001)$ and the authoritarian mother $(t=4.95$, $p<0.001)$ significantly predicted students' nonpsychological problems. The permissive father style was also a significant predictor in this model $(t=4.93, p<0.001)$. The other three parenting styles did not reach significance.

Table 2

Zero-Order Correlations for the Model's Variables $(\mathbf{N}=\mathbf{2 5 6 2})$

\begin{tabular}{lllllllll}
\hline Variable & PF & PM & VF & VM & NF & NM & PS-P & NON-P \\
\hline PF & 1 & 0.75 & 0.11 & 0.02 & 0.23 & 0.31 & 0.08 & 0.14 \\
PM & & 1 & 0.04 & 0.05 & 0.22 & 0.30 & 0.06 & 0.09 \\
VF & & & 1 & 0.57 & 0.09 & 0.13 & -0.14 & -0.12 \\
VM & & & & 1 & 0.16 & 0.09 & -0.13 & -0.09 \\
NF & & & & & 1 & 0.64 & 0.06 & 0.08 \\
NM & & & & & & 1 & 0.10 & 0.14 \\
PS-P & & & & & & 1 & 0.57 \\
NON-P & & & & & & & 1
\end{tabular}

Note. $\mathrm{PF}=$ permissive father; $\mathrm{PM}=$ permissive mother; $\mathrm{VF}=$ authoritative father; $\mathrm{VM}=$ authoritative mother; $\mathrm{NF}=$ authoritarian father; $\mathrm{NM}=$ authoritarian mother; $\mathrm{PS}-\mathrm{P}=$ psychological problems; $\mathrm{NON}=$ Non-Psychological problems

All values are significant $(p<.01)$ except $r=0.04(p<.05)$ and a non-significant $r=0.02$. 
Table 3

\begin{tabular}{llccccc}
\multicolumn{7}{c}{ Results of the Multiple Regression Models to Predict Students' Problems Using Parenting Styles } \\
\hline \multicolumn{1}{c}{ Model } & B & S.E & $\beta$ & t & sig & $\mathrm{R}^{2}$ \\
\hline Psychological Problems & & & & & & \\
Constant & 2.47 & 0.12 & - & 20.05 & 0.000 & \\
Authoritarian mother & 0.10 & 0.02 & 0.09 & 3.59 & 0.000 & \\
Authoritative mother & -0.08 & 0.03 & -0.07 & -2.98 & 0.003 & 0.041 \\
Permissive mother & 0.00 & 0.03 & 0.00 & 0.08 & 0.934 & \\
Authoritarian father & 0.01 & 0.02 & 0.01 & 0.54 & 0.583 & \\
Authoritative father & -0.11 & 0.02 & -0.11 & -4.55 & 0.000 \\
Permissive father & 0.05 & 0.03 & 0.05 & 1.79 & 0.073 & \\
Non-Psychological Problems & & & & & & \\
Constant & 1.75 & 0.13 & - & 13.41 & 0.000 & \\
Authoritarian mother & 0.14 & 0.03 & 0.13 & 4.95 & 0.000 & \\
Authoritative mother & -0.04 & 0.03 & -0.03 & -1.31 & 0.188 & 0.053 \\
Permissive mother & -0.05 & 0.03 & -0.05 & -1.64 & 0.101 & \\
Authoritarian father & -0.01 & 0.02 & -0.01 & -0.40 & 0.684 & \\
Authoritative father & -0.13 & 0.02 & -0.12 & -5.12 & 0.000 & \\
Permissive father & 0.17 & 0.03 & 0.15 & 4.93 & 0.000 & \\
\hline
\end{tabular}

\section{Discussion}

The researcher sought to expand the literature related to university students' perceptions of parenting styles and university life adjustment by examining the effects of parenting styles on students' levels of psychological and nonpsychological problems among a sample of 2562 undergraduate students enrolled in Sul$\tan$ Qaboos University in the Sultanate of Oman.

The findings of the study are consistent with earlier research (Aldhafri \& Al-Harthy, 2016) that proves the association between university students' perceptions of their parents' parenting styles and these students' outcomes and characteristics. The correlational analyses as well as the regression models support the existence of these connections.

Based on Cohen's criteria of size effects (1988), the two regression models showed small effects of parenting styles by explaining $5 \%$ of variance in the university students' feelings of psychological and non-psychological problems. Self-related variables may represent possible contributors to university students' liveswhich may count for the variance in the levels of problems and challenges they face during university years.

The results showed variation in the effects of each parenting styles on the development of university students' psychological and nonpsychological problems. Consistent with previous studies, the authoritative parenting styles seem to be essential in the positive chil- dren's outcomes as it related negatively to the levels of students' problems. Research in parenting styles worldwide almost all agree about the important role of authoritative parenting styles in supporting positive children's characteristics and eliminating negative ones (Driscoll, Russell, \& Crockett, 2008; Lohaus, Vierhaus, \& Ball, 2009).

In contrast, less and limited support of the effects of the permissive parenting styles are found in the current study. Only permissive father parenting style was found to significantly influence students' perceptions of nonpsychological problems. The results show that students' levels of non-psychological problems increase in families where fathers adopt permissive parenting style. Even though the permissive style (both for father and mother) correlated significantly and positively with the two types of problems in the simple correlation coefficients, their effects in the regression model were not significant in the first regression equation that predicted students' psychological problems and partially significant for fathers in the second equation that predicted students' non-psychological problems.

\section{Conclusion}

Finally, the authoritarian mother seems to be influential in predicting the two types of problems faced by the study sample. The findings suggest negative effects of this style on children's development. This style, however, does not seem to be critical for fathers as its effects were not significant in both regression equations. In a father oriented community, one 
may expect that authoritarian fathers have an important role in the development of children across different life stages. The non-significant effects of authoritarian father in both equations may represent some ongoing changes in the Omani family structure with the increasing role of women compared to men. With increasing levels of education and income for women, Omani mothers have became more influential in directing children's behavior and influencing their development. The significant results found for maternal styles in predicting university students' problems indicate that the effect of authoritarian mothers continue up to university years and are not limited to school years. In addition, authoritarian parenting style effects on children's development have been controversial in terms of being positive or negative; researchers examining collective cultures tend to find positive effects of authoritarian parenting styles compared to those examining individualistic cultures (Cheah, Leung, Tahseen, \& Schultz, 2009; Dwairy, \& Menshar, 2006).

The current findings suggest different direction in a collectivist culture like Oman and may reflect some developmental or cultural changes in the children-parent perceptions and interactions. Future research may examine this hypothesis by looking at the connections between parenting styles and children's problems in a mix of collectivist and individualist contexts using a cross-cultural research design. The inclusion of children from different ages may shed light on possible developmental changes in children's perceptions of parenting styles as children grow up.

The findings also support the importance of measuring parenting styles separately for fathers and mothers. While two maternal parenting styles were significant in predicting children's psychological problems, only one paternal style was significant. This pattern was the opposite when it comes to predicting nonpsychological problems where two paternal and one maternal style were significant. The strength of these effects also varied across the significant correlations. These differences can be viewed through psychological characteristics of mothers in Omani society which allow them to have more influence than fathers on their children's psychological problems.

The low levels of permissive parenting styles and the high levels of authoritative and au- thoritarian styles reported by these Omani students indicate that Omani parents practice demanding style (that need to be high in both authoritative and authoritarian styles) as conceptualized by Boumrind (1991). The same pattern was found to be common earlier in Omani society by Aldhafri (2011). This may relate to the nature of the Omani society that is common in the Arabic culture (as a collectivist culture) where obedience of parents and respect of high figures of the family are highly advocated early in childhood (Abu-Hilal, Aldhafri, Albahrani, \& Kamali, 2016).

Future research needs to examine other possible factors that critically influence university students' perceptions and development of psychological and non-psychological problems. This research needs to examine selfrelated variables that may contribute largely to students' ability to adapt positively to the university life and challenges. Examples include, but not limited to, academic identity, selfregulated strategies, academic self-efficacy, self-concept and esteem, emotional intelligence, adaptation skills, and general life skills.

\section{References}

Abu-Hilal, M., Aldhafri, S., \& Albahrani, M., Kamali, M. (2016). The Arab culture and the Arab self: Emphasis on gender. In R. King \& A. Bernardo (Eds.), The Psychology of Asian Learners (pp. 125-138). Singapore: Springer Singapore.

Al-Darmaki, F. (2003). Attitudes towards seeking professional psychological help: What really counts for United Arab Emirates University students? Social Behavior and Personality: An International Journal, 31 (5), 497-508. doi:10.2224/sbp.2003.31.5.497.

Al-Darmaki, F. (2011). Problems and preferences for source of help among United Ar$\mathrm{ab}$ Emirates university students. International Journal of Advice and counseling, 33, $293-308$.

Aldhafri, S. (2011). Self-efficacy and physical self-concept as mediators of parenting influence on adolescents' adjustment and wellbeing. Journal of Psychology in Africa, 21(4), 511-520.

Aldhafri, S., \& Al-Harthy, I. (2016). Undergraduate Omani students' identity and perceptions of parenting Styles. Review of 
European Studies, 8(2), 114-123. doi:10.5539/res.v8n2p114

Alkharusi, H., Aldhafri, S., Kazem, A., Alzubiadi, A., \& Al-Bahrani, M. (2011). Development and Validation of a Short Version of the Parental Authority Questionnaire. Social Behavior and Personality, 39, 1193-1208.

Asberg, K. (2013). Hostility/anger as a mediator between college students' emotion regulation abilities and symptoms of depression, social anxiety, and generalized anxiety. The Journal of Psychology, 147(5), 469-490.

Baldwin, D., McIntyre, A., \& Hardaway, E. (2007). Perceived parenting styles on college students' optimism. College Student Journal, 41(3), 550-557.

Baumrind, D. (2005). Patterns of parental authority and adolescent autonomy. New Directions for Child and Adolescent Development, 2005 (108), 61-69.

Baumrind, D. (1991). The influence of parenting style on adolescent competence and substance use. Journal of Early Adolescence, 11(1), 56-95.

Benton, S., Robertson, J., Tseng, W., Newton, F., \& Benton, S. (2003). Changes in counseling center client problems across 13 years. Professional Psychology: Research and Practice, 34(1), 66-72. DOI: 10.1037/0735-7028.34.1.66

Buri, J. (1991). Parental Authority Questionnaire. Journal of Personality Assessment, 57(1), 110-119.

Chandler, L., \& Gallagher, R. (1996). Developing a taxonomy for problems seen at a university counseling center. Measurement and Evaluation in Counseling and Development, 29, 4-12.

Cheah, C., Leung, C., Tahseen, M., \& Schultz, D. (2009). Authoritative parenting among immigrant Chinese mothers of preschoolers. Journal of Family Psychology, 23 (3), 311320 .

Conrade, G., \& Ho, R. (2001). Differential parenting styles for fathers and mothers. Australian Journal of Psychology, 35(1), 29-35.

Driscoll, A., Russell, S., \& Crockett, L. (2008). Parenting styles and youth well-being across immigrant generations. Journal of FamilyIssues, 29 (2), 185-209.
Dwairy, D., \& Menshar, K. (2006). Parenting style, individuation, and mental health of Egyptian adolescents. Journal of Adolescence, 29 (1), 103-117.

Estep, H., \&Olson, J. (2011). Parenting style, academic dishonesty, and infidelity in college students. College Students Journal, 45(4), 830-838.

Gracia, E., \& Gracia, F. (2009). Is always authoritative the optimum parenting style? Evidence from Spanish families. Adolescence, 44(173), 101-132.

Grundman, J. (2010). Does Parenting style predict identity and emotional outcomes in emerging adulthood. College Journal of Gustavus Undergraduate Psychology, 6, https://gustavus.edu/psychology/docum ents/JennyGrundman.pdf

Gryckowski, M., Jordan, S., \& Mercer, S. (2010). Differential relations between mothers' and fathers' parenting practices and child externalizing behavior. Journal of Child and Family Studies, 19, 539-546.

Ishak, Z., Low,S., Lau,P. (2012). Parenting style as a moderator of students' academic achievement. Journal of Science Educational Technology, 21, 487-493.

Joshi, A., Ferris, J., Otto, A., \& Regan, P. (2003). Parenting styles and academic achievement in college students. Psychological Reports, 93, 823-828.

Love, K.,\& Thomas, D. (2014). Parenting styles and adjustment outcomes among college students. Journal of College Student Development, 55(2). 139-150.

Lohaus, A., Vierhaus, M., \& Ball, J. (2009). Parenting styles and health-related behavior in childhood and early adolescence results of a longitudinal study. Journal of Early Adolescence, 29(4), 449-475.

Maddahi, M., Javidi, N., Samadzadeh, M., \&Amini, M. (2012). The study of relationship between parenting styles and personality dimensions in sample of college students. Indian Journal of Science and Technolo$g y, 5(9), 3332-3336$.

Mckinney, C., Milone, M., \& Renk, K. (2011). Parenting and late adolescent emotional adjustment: mediating effect of discipline and gender. Child Psychiatry and Human 
Development, 42, 463-481. doi: $10.1007 /$ s10578-011-0229-2.

Mckinney, C., \& Renk, K. (2008). Differential parenting between mothers and fathers: Implications for late adolescents. Journal of Family Issues, 29(6), 806-827. doi: $10.1177 / 0192513 \times 07311222$

Nguyen, P., \& Chenung, M. (2009). Parenting styles as perceived by Vietnamese American adolescents. Child EAdolescent Social Work Journal, 26, 505-518. doi 10.1007/s10560-009-0182-0

Pedrelli, P., Borsari, B., Palm, K., Dalton, E., \& Fava, M. (2013). Combined MI + CBT for depressive symptoms and binge drinking among young adults: Two case studies. Journal of Cognitive Psychotherapy, 27(3), 235257.

Reynolds, A. (2013). College student concerns: Perceptions of student affairs practitioners. Journal of College Student Development, 54(1), 98-105.

Rinaldi, C., \& Howe, N. (2012). Mothers' and fathers' parenting styles and associations with toddlers' externalizing, internalizing, and adaptive behaviors. Early Childhood Research Quarterly, 27(2), 266-273.

Wagner, M., \& Rhee, Y. (2013). Stress, sleep, grief: Are college students receiving information that interests them? College Student Journal, 24-33. 